\title{
Errata to (Nearly) Round-Optimal Black-Box \\ Constructions of Commitments Secure against Selective Opening Attacks
}

\author{
David Xiao \\ LIAFA \\ CNRS and Université Paris Diderot - Paris 7 \\ dxiao@liafa.univ-paris-diderot.fr
}

\begin{abstract}
Several proofs initially presented by the author 2 were shown to be incorrect in a recent work of Ostrovsky et al. [1]. In this notice we summarize the errors and summarize the current state of the art after taking into account the errors and subsequent work.
\end{abstract}

In TCC 2011 the author claimed several results about nearly round-optimal black-box constructions of commitments secure against selective opening attacks [2]. It was later shown by Ostrovsky et al. [1] (a proceedings version appears in the current volume), that several of the proofs in 2 contained errors. Here we restate the errors discovered by Ostrovsky et al. 1, and we summarize what remains true from [2], as well as the current state of the art in light of the revised theorems from [2] and subsequent work including [1].

Errors in [2]: (for details, we refer the reader to [1]

1. The proof of Theorem 1, which claimed several nearly round-optimal blackbox constructions, is incorrect as presented there. This is due to problems with the hiding and binding properties of the constructions presented there.

2. Items 1 and 2 of Theorem 2, which claimed to rule out selective-opening secure black-box constructions of 3-round parallel computational binding and hiding commitments and 4-round parallel statistically binding commitments, are incorrect. This is due to an incorrect implicit assumption that the sender sends the last message in the commit phase.

Unaffected results: the proofs of the following theorems from [2] remain valid:

1. Item 3 of Theorem 2, stating that one can build constant-round stand-alone statistically hiding commitments in a black-box way using constant-round statistically binding parallel selective-opening secure commitments.

2. Corollary 1, stating that there is no black-box construction using one-way permutations to build constant-round statistically binding parallel selectiveopening secure commitments.

3. Theorem 3, stating there exist no black-box constructions for constant-round receiver public-coin protocols and or perfect binding protocols.

Item 4 of Theorem 2 of 2 regarding fully concurrent selective-opening security also remains valid, but this is superseded by the results of [1] (see below).

A. Sahai (Ed.): TCC 2013, LNCS 7785, pp. 721-722, 2013.

(C) International Association for Cryptologic Research 2013 
Revised results: The following weakened statement of Theorem 2, Items 1 and 2 of [2] holds, using the original proof except removing the incorrect implicit assumption that the sender sends the last message in the commit phase:

Theorem (Revision of Theorem 2 of [2]). There exist no black-box constructions of commitments that are parallel selective-opening secure with 2 rounds and that are computationally binding and hiding, or with 3 rounds and that are statistically binding.

The author was also able to give a different proof of Item 2 of Theorem 1 of [2, which claimed a black-box construction of $(t+3)$ statistically-binding parallel selective-opening secure commitments assuming $t$-round stand-alone statistically hiding commitments, but this is superseded by the results of [4] (see below).

State of the art: For parallel selective opening security, Ostrovsky et al. [1] and subsequent work of the author [4] gave the following black-box constructions:

1. 3-round computationally binding and hiding commitments, assuming appropriate stand-alone trapdoor commitment schemes [1] (this is optimal by the revised theorem above).

2. $(t+2)$-round statistically binding commitments, assuming the existence of stand-alone $t$-round statistically hiding commitments [4]. (For the case $t=2$ this is optimal by the above revised theorem.)

Ostrovsky et al. [1] also give other constructions with different round complexities under weaker assumptions and/or allowing interactive decommitment.

For concurrent security, it was proved in [1] that no secure black-box constructions exist with fully concurrent selective-opening security, although their constructions (including their 3-round construction) are secure in a model they term concurrent-with-barrier. We refer the reader to [1] for details.

Revised Manuscript: a revised (unrefereed) manuscript [3] is available on the Cryptology ePrint archive containing the valid results from [2].

\section{References}

[1] Ostrovsky, R., Rao, V., Scafuro, A., Visconti, I.: Revisiting Lower and Upper Bounds for Selective Decommitments. Cryptology ePrint Archive, Report 2011/536 (2011), http://eprint.iacr.org/

[2] Xiao, D.: (Nearly) Round-Optimal Black-Box Constructions of Commitments Secure against Selective Opening Attacks. In: Ishai, Y. (ed.) TCC 2011. LNCS, vol. 6597, pp. 541-558. Springer, Heidelberg (2011)

[3] Xiao, D.: On the round complexity of black-box constructions of commitments secure against selective opening attacks. Technical Report 2009/513, Cryptology ePrint Archive (2012)

[4] Xiao, D.: Round-Optimal Black-Box Statistically Binding Selective-Opening Secure Commitments. In: Mitrokotsa, A., Vaudenay, S. (eds.) AFRICACRYPT 2012. LNCS, vol. 7374, pp. 395-411. Springer, Heidelberg (2012) 